\title{
Stereoelectroencephalography-guided radiofrequency thermocoagulation in the epileptogenic zone: a retrospective study on 89 cases
}

\author{
Massimo Cossu, MD, ${ }^{1}$ Dalila Fuschillo, MD, ${ }^{2}$ Giuseppe Casaceli, MD, ${ }^{1,3}$ Veronica Pelliccia, MD, ${ }^{1,3}$ \\ Laura Castana, MD, ${ }^{1}$ Roberto Mai, MD, ${ }^{1}$ Stefano Francione, MD, ${ }^{1}$ Ivana Sartori, MD, ${ }^{1}$ \\ Francesca Gozzo, MD, ${ }^{1}$ Lino Nobili, MD, ${ }^{1,4}$ Laura Tassi, MD,, Francesco Cardinale, MD, ${ }^{1}$ and \\ Giorgio Lo Russo, MD'
}

\begin{abstract}
${ }^{1}$ Epilepsy Surgery Center and ${ }^{4}$ Center of Sleep Medicine, Department of Neuroscience, Niguarda Hospital, Milan; ${ }^{2}$ Section of Neurosurgery, Department of Neurosciences and of Sense Organs, University of Milan; and ${ }^{3}$ Department of Neuroscience, University of Parma, Italy
\end{abstract}

\begin{abstract}
OBJECT Radiofrequency thermocoagulation (RF-TC) of presumed epileptogenic lesions and/or structures has gained new popularity as a treatment option for drug-resistant focal epilepsy, mainly in patients with mesial temporal lobe epilepsy. The role of this minimally invasive procedure in more complex cases of drug-resistant epilepsy, which may require intracranial electroencephalographic evaluation, has not been fully assessed. This retrospective study reports on a case series of patients with particularly complex focal epilepsy who underwent stereoelectroencephalography (SEEG) evaluation with stereotactically implanted multicontact intracerebral electrodes for the detailed identification of the epileptogenic zone (EZ) and who received RF-TC in their supposed EZ (according to SEEG findings).
\end{abstract}

METHODS Eighty-nine patients ( 49 male and 40 female; age range 2-49 years) who underwent SEEG evaluation and subsequent RF-TC of the presumed EZ at the authors' institution between January 2008 and December 2013 were selected. Brain MRI revealed structural abnormalities in 43 cases and no lesions in 46 cases. After SEEG, 67 patients were judged suitable for resective surgery (Group 1), whereas surgery was excluded for 22 patients (Group 2). Thermocoagulation was performed in each of these patients by using the previously implanted multicontact recording electrodes and delivering RF-generated currents to adjacent electrode contacts.

RESULTS The mean number of TC sites per patient was $10.6 \pm 7.2$ (range 1-33). Sustained seizure freedom occurred after TC in 16 patients (18.0\%) (13 in Group 1 and 3 in Group 2). A sustained worthwhile improvement was reported by 9 additional patients (10.1\%) (3 in Group 1 and 6 in Group 2). As a whole, 25 patients (28.1\%) exhibited a persistent significant improvement in their seizures. More favorable results were observed in patients with nodular heterotopy ( $p$ $=0.0001389$ ), those with a lesion found on MRI (not significant), and those with hippocampal sclerosis (not significant). Other variables significantly correlated to seizure freedom were the patient's age $(p=0.02885)$ and number of intralesional TC sites $(p=0.0271)$. The patients in Group 1 who did not benefit at all (21 patients) or who experienced only a transient benefit (30 patients) from TC underwent microsurgical resection of their EZ. Thermocoagulation was followed by severe permanent neurological deficits in 2 patients (an unexpected complex neuropsychological syndrome in one patient and an expected and anticipated permanent motor deficit in the other).

CONCLUSIONS This study provides evidence that SEEG-guided TC in the EZ may be a treatment option for particularly complex drug-resistant focal epilepsy that requires invasive evaluation. A small subset of patients who achieve seizure freedom or worthwhile improvement may avoid open surgery or take advantage of an otherwise unexpected treatment if resection is not an option. Patients with epileptogenic nodular heterotopy are probably ideal candidates for this treatment.

http://thejns.org/doi/abs/10.3171/2014.12.JNS141968

KEY WORDS stereo-EEG; radiofrequency thermocoagulation; epilepsy surgery; epileptogenic zone; seizure outcome

ABBREVIATIONS EEG = electroencephalography; EZ = epileptogenic zone; FCD = focal cortical dysplasia; MTLE = mesial temporal lobe epilepsy; RF-TC = radiofrequency thermocoagulation; SEEG = stereoelectroencephalography.

SUBMITTED August 28, 2014. ACCEPTED December 8, 2014.

INCLUDE WHEN CITING Published online June 19, 2015; DOI: 10.3171/2014.12.JNS141968.

DISCLOSURE Dr. Cardinale is a consultant for Renishaw Mayfield SA, the manufacturer of Neuromate, and a former consultant for Medtronic, the manufacturer of the O-arm device. The other authors report no conflict of interest concerning the materials or methods used in this study or the findings specified in this paper. 
$\mathrm{S}$ TEREOTACTIC destruction of presumed epileptogenic cortical areas or lesions has been advocated in the past as a treatment option for patients with drug-resistant focal epilepsy. Mesial temporal structures were the preferred targets in several earlier studies, which reported not particularly encouraging results related to seizures (exhaustive reviews have been performed by Parrent and Blume ${ }^{20}$ and by Guénot et al. ${ }^{12}$ ). With the advent of modern imaging techniques and the increasing accuracy of stereotactic targeting, interest in stereotactic ablative procedures, specifically radiofrequency thermocoagulation (RF-TC), has recently gained new popularity. Promising results in temporal lobe epilepsy ${ }^{14,16,17}$ and in epilepsies symptomatic of certain malformative lesions ${ }^{15,21,23}$ have been reported. Nevertheless, the role of this minimally invasive procedure in patients with more complex drug-resistant focal epilepsy, which may require invasive electroencephalography (EEG) evaluation, and in those who are not optimal candidates for resective surgery is still unclear.

Stereoelectroencephalography (SEEG), the clinical use of which in the presurgical evaluation of epileptic patients was introduced in the last century by a group at SainteAnne Hospital in Paris, France, is a powerful tool for defining the spatial and chronological evolution of an ictal discharge. ${ }^{1}$ SEEG is currently used in several centers to better investigate patients with more complex epilepsy, whose candidacy for surgical resection of their epileptogenic zone (EZ) is not adequately supported by noninvasive evaluation. ${ }^{3,5,10}$ In addition to recording brain electrical activity, SEEG enables accurate functional mapping of cortical and subcortical eloquent structures by using intracerebral electrical stimulations. Electrical stimulations may also trigger habitual electroclinical ictal manifestations and provide a better definition of the organization of the EZ.19

Intracerebral electrodes used for SEEG recording have also been used to produce RF-TC in epileptic patients whose EZ had been identified by SEEG recording., ${ }^{42,13}$ SEEG-guided RF-TC in the EZ has been found to be a treatment option in patients not suitable for resective surgery, particularly in those with malformations of cortical development in eloquent areas or with bilateral lesions. ${ }^{12}$ In a recent retrospective study, sustained seizure freedom in 4 of 5 patients who were treated with SEEG-guided RF$\mathrm{TC}$ of epileptogenic nodular gray matter heterotopy was reported. ${ }^{6}$

In the present retrospective study, we report on the experience of our center in a consecutive series of patients with both lesional and nonlesional drug-resistant focal epilepsy who received SEEG evaluation followed by RF-TC in their supposed EZ.

\section{Methods}

We retrospectively reviewed our clinical database in a search for patients who underwent an SEEG evaluation and SEEG-guided RF-TC in their EZ. Each patient or his or her guardians gave informed consent to both implantation of the electrodes for SEEG and subsequent RF-TC procedures. This study received the approval of the local ethical committee.

\section{Stereoelectroencephalography}

Indications for SEEG evaluation and the technique for implanting the electrodes for SEEG have been detailed elsewhere. ${ }^{3,5}$ Briefly, SEEG was indicated when a tailored noninvasive presurgical evaluation failed to clearly define the patient's EZ. For each patient, a customized arrangement of intracerebral electrodes was planned with the aim of verifying 1 or more working hypotheses based on the results of previous investigations. Each patient underwent brain MRI (Achieva 1.5 T, Philips Healthcare). Several sequences appropriate for SEEG planning were acquired; the essential 3D T1-weighted MRI scan was obtained in the sagittal plane and reformatted into axial slices with a $560 \times 560$ matrix, a $0.46 \times 0.46 \times 0.9-\mathrm{mm}$ voxel, and no interslice gap. Other structural MRI, functional MRI, diffusion tensor imaging, brain CT, or CT-PET scans were obtained when needed. Pertinent cerebral vessels were also imaged by using an O-arm 1000 system (Medtronic), which is a mobile cone-beam CT system that ensures 0.4 $\times 0.4 \times 0.8-\mathrm{mm}$ reconstructed voxels, to obtain $3 \mathrm{D}$ digital subtraction angiographic data. After coregistration of the acquired data sets to the 3D digital subtraction angiographic images, implantation of the SEEG electrodes was planned with the aim of accurately targeting the desired structures through avascular trajectories. Stereotactic electrode placement was performed with the patient under general anesthesia; a Talairach frame and a robotized passive tool-holder (Neuromate, Renishaw Mayfield SA) were used. A variable number of multicontact electrodes (Microdeep intracerebral electrodes, D08 [Dixi Medical], or Depth Electrodes Range 2069 [Alcis]) were placed and advanced to the desired targets, after screwing a hollow peg to the skull, and fixed by a plastic cap. After implantation, a final cone-beam CT data set was acquired by using the O-arm to verify the actual position of the electrodes. The T1-weighted 3D MR image was coregistered to this data set, which enabled us to accurately localize the electrodes with respect to the brain structures. After the surgical procedure, the patient was awakened, and intensive video-EEG monitoring began. Once data from an adequate number of spontaneous seizures were captured, a session of intracerebral electrical stimulations was performed. Both low-frequency ( $1 \mathrm{~Hz}$, pulse width $1-3 \mathrm{msec}$, delivered for 30 seconds) and high-frequency $(50 \mathrm{~Hz}$, pulse width $1 \mathrm{msec}$, delivered for 5 seconds) stimulations were delivered with the aim of reproducing entirely or in part the clinical ictal manifestations. Functional cortical and subcortical mapping 5 was then performed by recording clinical physiological manifestations induced by electrical stimulations and multimodal evoked potentials.

Once monitoring was completed, recorded material was reviewed and interpreted by the epileptologist with the aim of identifying the EZ as the area of ictal onset and early propagation of the ictal discharge. Therefore, each patient was labeled as suitable for resection (Group 1) or excluded from surgery (Group 2). Reasons for exclusion from surgery included an EZ that involved functionally critical areas, the resection of which would create unacceptable new neurological deficits; nonlocalizing SEEG findings (multifocal/bilateral ictal onset or poor definition of the EZ); and patient refusal to undergo resection. 


\section{Radiofrequency Thermocoagulation}

Radiofrequency thermocoagulation has been offered to every patient at our center undergoing SEEG monitoring since 2008. Only patients who agreed to receive this treatment underwent the procedure. Group 2 patients were openly informed that RF-TC was the only treatment option available.

Radiofrequency thermocoagulation was performed at the end of the recording period and before electrode removal. No anesthesia was used, which enabled clinical monitoring of the patient during the procedures.

Each thermocoagulative lesion was placed between a couple of contiguous electrode contacts. The contacts used for RF-TC were selected according to the presence of 1 or more of the following criteria: 1 ) their involvement in the onset of the ictal discharge, 2) an intralesional location, and/or 3) induction of habitual ictal clinical phenomena by their electrical stimulations.

If the selected contacts sampled a functionally critical area, as documented by stereoelectroencephalographic functional mapping (e.g., movement, speech, vision), they were excluded from treatment. Similarly, no coagulation was performed in proximity of vascular structures $(<2$ $\mathrm{mm}$ from selected contacts). Therefore, the original treatment plan could not be completely realized.

The 2 pertinent contacts were connected to RF lesiongenerator equipment (NeuroN50 and NeuroN100 [Stryker Leibinger] with Dixi Medical and Alcis electrodes, respectively), expressly modified for use with SEEG electrodes; because no local temperature monitoring is possible when using SEEG electrodes, the temperature feedback was excluded from the generator circuitry. We adopted the following parameters: current power progressively raised from 1.5 to $8.32 \mathrm{~W}$ within 60 seconds and current intensity (usually approximately $25 \mathrm{~mA}$ ) variable according to impedance. These parameters were defined specifically to increase the tissue temperature to $78^{\circ}-82^{\circ} \mathrm{C}$, which had been reported previously to induce a lesion around the selected contacts within $40-50$ seconds. , $^{42,13}$

At the end of the procedure, the electrodes could be removed, and the patients were discharged within 1-2 days. When possible, an MRI study was obtained at least 1 month after the procedure.

\section{Resective Surgery}

Patients from Group 1 who did not benefit from RF-TC (no benefit at all or transient benefit) subsequently underwent surgery after a variable period of follow-up. Resective microsurgery was conducted with the aim of removing the EZ as defined by SEEG evaluation.

\section{Seizure Outcomes and Statistical Analysis}

Post-RF-TC seizure outcomes were prospectively assessed at regular intervals by outpatient visits or telephone contacts. The patients were assigned to 1 of the following categories: seizure free (corresponding to Engel Class $\left.I^{8}\right)$, sustained worthwhile improvement, transient benefit (initial improvement and then return to baseline), or no benefit. Statistical evaluation of seizure outcomes was reserved for patients with a follow-up period of $\geq 12$ months after RF-TC (for those who received RF-TC as the sole treatment) and for patients who subsequently received resective surgery and whose unfavorable seizure outcome could be considered conclusive regardless of the time elapsed after RF-TC.

The following variables were considered: sex, epilepsyrelated antecedents, age at seizure onset, age at RF-TC, duration of epilepsy, seizure frequency, preoperative MRI results (lesional or nonlesional), extension of lesion in MR images, etiology of epilepsy (assessed by MRI or, for those who underwent surgery, histology), induction of seizures by low- or high-frequency intracerebral electrical stimulations, RF-TC performed on contacts through which stimulations induced ictal manifestations, contribution of SEEG to EZ localization (localizing or nonlocalizing), site of the EZ (temporal or extratemporal), site of RF-TC, total number of RF-TC sites, and number of RF-TC sites within the MRI-detected abnormality. Each variable was investigated for the chances of RF-TC to produce sustained seizure freedom (Engel Class I).

Furthermore, a separate analysis was performed for patients who underwent surgery to evaluate whether significant differences in postresection seizure outcome occurred in patients who had and in those who had not experienced a transient benefit on seizures after RF-TC. For this analysis, only patients with a minimum postresection follow-up time of 12 months were included.

For bivariate analysis, the Kruskal-Wallis rank-sum test was used to analyze numerical variables, and Fisher's 2 -tailed exact test was performed to analyze categorical (binomial or multinomial) variables. A $p$ value of $<0.05$ was considered evidence of findings not attributable to chance. Statistical analysis was performed using R 3.0.3 (R Development Core Team [http://www.R-project.org/]).

\section{Results}

We selected 89 patients who received RF-TC after SEEG evaluation; 49 were male and 40 were female. In each case, the seizures were drug resistant, because $\geq 2$ antiepileptic drugs in monotherapy or polytherapy at maximum tolerated doses had failed to produce sustained seizure freedom. The mean age at treatment was 26.7 years (SD 11.3; range 2-49 years), the mean age at seizure onset was 11.3 years (SD 9.8; range 0-39 years), and the mean duration of epilepsy was 15.4 years (SD 9.5; range 1-43 years).

Preoperative brain MRI results were negative (not lesional) in 46 patients, whereas they showed a structural lesion (lesional) in 43 patients. The lesion was monolateral in 35 patients (unilobar in 29 patients and multilobar in 6 patients), and 8 patients harbored bilateral lesions.

\section{Stereoelectroencephalography}

The main features of the SEEG evaluations are reported in Table 1. On the basis of SEEG recordings, $67 \mathrm{pa-}$ tients were judged suitable for resective surgery (Group 1), and surgery was excluded for 22 patients (Group 2). The reasons for exclusion from resective surgery were an EZ that involved highly eloquent areas (4 patients) and nonlocalizing SEEG results (13 patients: 3 with multifocal/ 
TABLE 1. Main features of stereoelectroencephalographic evaluations

\begin{tabular}{lc}
\hline \multicolumn{1}{c}{ Feature (no. of pts) } & Value $^{*}$ \\
\hline $\begin{array}{l}\text { No. of implanted multicontact electrodes per pt } \\
(\mathrm{n}=89)\end{array}$ & $13.9 \pm 2.6(4-21)$ \\
\hline $\begin{array}{l}\text { No. of multicontact electrodes reaching an MRI } \\
\quad \text { lesion/pt }(\mathrm{n}=43)\end{array}$ & $3.8 \pm 3.2(0-15)$ \\
$\begin{array}{l}\text { No. of single contacts reaching an MRI lesion/ } \\
\text { pt }(\mathrm{n}=43)\end{array}$ & $17.0 \pm 15.9(0-69)$ \\
\hline Pts w/ seizures elicited by LFES & 53 \\
\hline Pts w/ seizures elicited by HFES & 74 \\
\hline
\end{tabular}

HFES = high-frequency electrical stimulations; LFES = low-frequency electrical stimulations; $\mathrm{pt}=$ patient.

* Values are mean \pm SD (range) or number.

bilateral seizure onset and 10 with insufficient definition of the EZ). Five patients refused resective surgery and accepted RF-TC as their only treatment option.

\section{RF-TC, Seizure Outcome, and Statistical Analysis}

Pertinent data on the RF-TC procedures are reported in Table 2.

Postcoagulation MRI was obtained in 28 patients an average of 6.7 months (SD 3.7) after the procedure (Fig. 1). The mean volume of single RF-TC lesions (between 2 contiguous contacts), as measured on postcoagulation MR images, was $85.4 \mathrm{~mm}^{3}$ (SD 31.1).

Table 3 reports the outcome on seizures after RF-TC in the whole series and in selected subgroups of patients.

The preimplantation MRI for the 16 seizure-free patients revealed no lesions in 5 patients and a lesion in 11 patients. In the lesional cases, MRI showed nodular heterotopies in 8 patients (bilateral in 2) and hippocampal sclerosis in 3 patients. In the 9 patients with sustained worthwhile improvement, the MRI revealed no lesions in 5 patients, hippocampal sclerosis in 2 patients, focal cortical dysplasia (FCD) in 1 patient, and glial scarring in 1 patient.

Statistical analysis, aimed at revealing factors that significantly influence seizure outcome after RF-TC, was performed on 82 patients, including the 51 with a definite unfavorable outcome after RF-TC and who subsequently underwent surgery and the 31 who received RF-TC as their sole treatment and had a postprocedural follow-up time of $\geq 12$ months. Nodular heterotopy as the etiology

TABLE 2. Main features of RF-TC procedures

\begin{tabular}{lc}
\hline \multicolumn{1}{c}{ Feature (no. of pts) } & \multicolumn{1}{c}{ Value $^{*}$} \\
\hline RF-TC procedures/pt ( $\mathrm{n}=89$ ) & $10.6 \pm 7.2(1-33)$ \\
\hline $\begin{array}{l}\text { RF-TC procedures in the MRI lesion per pt ( } \\
\quad=43)\end{array}$ & $6.3 \pm 7.0(0-30)$ \\
\hline $\begin{array}{l}\text { Pts w/ RF-TC of regions in which LFES induced } \\
\text { habitual seizures }\end{array}$ \\
$\begin{array}{l}\text { Pts w/ RF-TC of regions in which HFES induced } \\
\text { habitual seizures }\end{array}$ \\
\hline
\end{tabular}

*Values are mean $\pm \mathrm{SD}$ (range) or number.
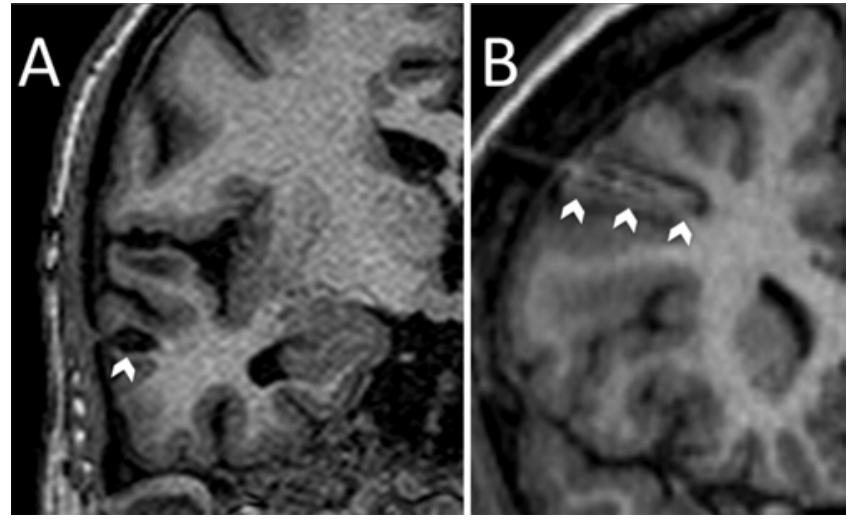

FIG. 1. A: A single RF-TC was performed between 2 adjacent contacts in the right middle temporal gyrus (arrowhead). This coronal T1-weighted MR image reveals an ovoid-shaped hypointense lesion (long axis 6 $\mathrm{mm}$, maximal thickness approximately $3.5 \mathrm{~mm}$ ). B: Six adjacent RF-TC procedures were performed between 7 contiguous electrode contacts in the right middle frontal gyrus (arrowheads). The long axis of the resulting lesion measures $20 \mathrm{~mm}$, and the maximal thickness is approximately $3.5 \mathrm{~mm}$.

$(\mathrm{p}=0.0001389)$, patient age $(\mathrm{p}=0.02885)$, and number of intralesional TC sites $(\mathrm{p}=0.0271)$ were factors with a significant correlation with seizure outcome.

The 51 patients in Group 1 who did not benefit at all ( 21 patients) or who experienced only a transient benefit (30 patients) from RF-TC underwent microsurgical resection

TABLE 3. Outcome of seizures after RF-TC in the entire series and in selected subgroups*

\begin{tabular}{lcccc}
\hline & & \multicolumn{3}{c}{ Outcome (no. [\%]) } \\
\cline { 3 - 5 } \multicolumn{1}{c}{ Group } & $\begin{array}{c}\text { No. of } \\
\text { Pts }\end{array}$ & $\begin{array}{c}\text { Seizure } \\
\text { Free }\end{array}$ & $\begin{array}{c}\text { Sustained } \\
\text { Worthwhile } \\
\text { Improvement }\end{array}$ & $\begin{array}{c}\text { Transient/No } \\
\text { Benefit }\end{array}$ \\
\hline All pts & 89 & $16(18.0)$ & $9(10.1)$ & $64(71.9)$ \\
\hline MRI & & & & \\
Lesional & 43 & $11(25.6)$ & $4(9.3)$ & $28(65.1)$ \\
$\quad$ Nonlesional & 46 & $5(10.9)$ & $5(10.9)$ & $36(78.2)$ \\
\hline SEEG & & & & \\
Localizing & 76 & $14(18.4)$ & $7(9.2)$ & $55(72.4)$ \\
$\quad$ Nonlocalizing & 13 & $2(15.4)$ & $2(15.4)$ & $9(69.2)$ \\
\hline Site of EZ & & & & \\
$\quad$ Temporal & 15 & $2(13.3)$ & $3(20.0)$ & $10(66.7)$ \\
Extratemporal & 74 & $14(18.9)$ & $6(8.1)$ & $54(73.0)$ \\
\hline Etiology† & & & & \\
HS & 6 & $3(50)$ & $2(33.3)$ & $1(16.7)$ \\
NH & 12 & $8(66.7)$ & $0(0)$ & $4(33.3)$ \\
FCD & 11 & $0(0)$ & $1(9.1)$ & $10(90.9)$ \\
Other & 60 & $5(8.3)$ & $6(10)$ & $49(81.7)$ \\
\hline Group 1 & 67 & $13(19.4) \ddagger$ & $3(4.5) \ddagger$ & $51(76.1) \S$ \\
Group 2 & 22 & $3(13.6)$ & $6(27.3)$ & $13(59.1)$ \\
\hline
\end{tabular}

$\mathrm{HS}=$ hippocampal sclerosis; $\mathrm{NH}=$ nodular heterotopy.

* See text for definitions of Groups 1 and 2. Percentages are calculated across rows.

$\dagger$ Etiology as revealed by histological examination in patients who underwent surgery or according to MRI findings in patients who received only RF-TC. $\ddagger$ Surgery recommended after SEEG and not performed after effective RF-TC. $\S$ Submitted to resection after ineffective RF-TC. 
of their EZ based on SEEG results. Among 22 of these patients with $\geq 12$ months of postresection follow-up, no significant correlation was observed between a transient improvement after RF-TC and the chances to achieve a postresection Class I outcome. Nevertheless, a higher proportion of postresection Class I outcomes was found among patients who experienced a transient benefit from
RF-TC than in those who did not respond at all to RF-TC (70\% and 58\%, respectively).

An illustrative case is detailed in the Fig. 2 legend.

\section{Etiology of Epilepsy}

The etiologies of epilepsy in the 89 studied patients, as revealed by histological examination in patients who un-

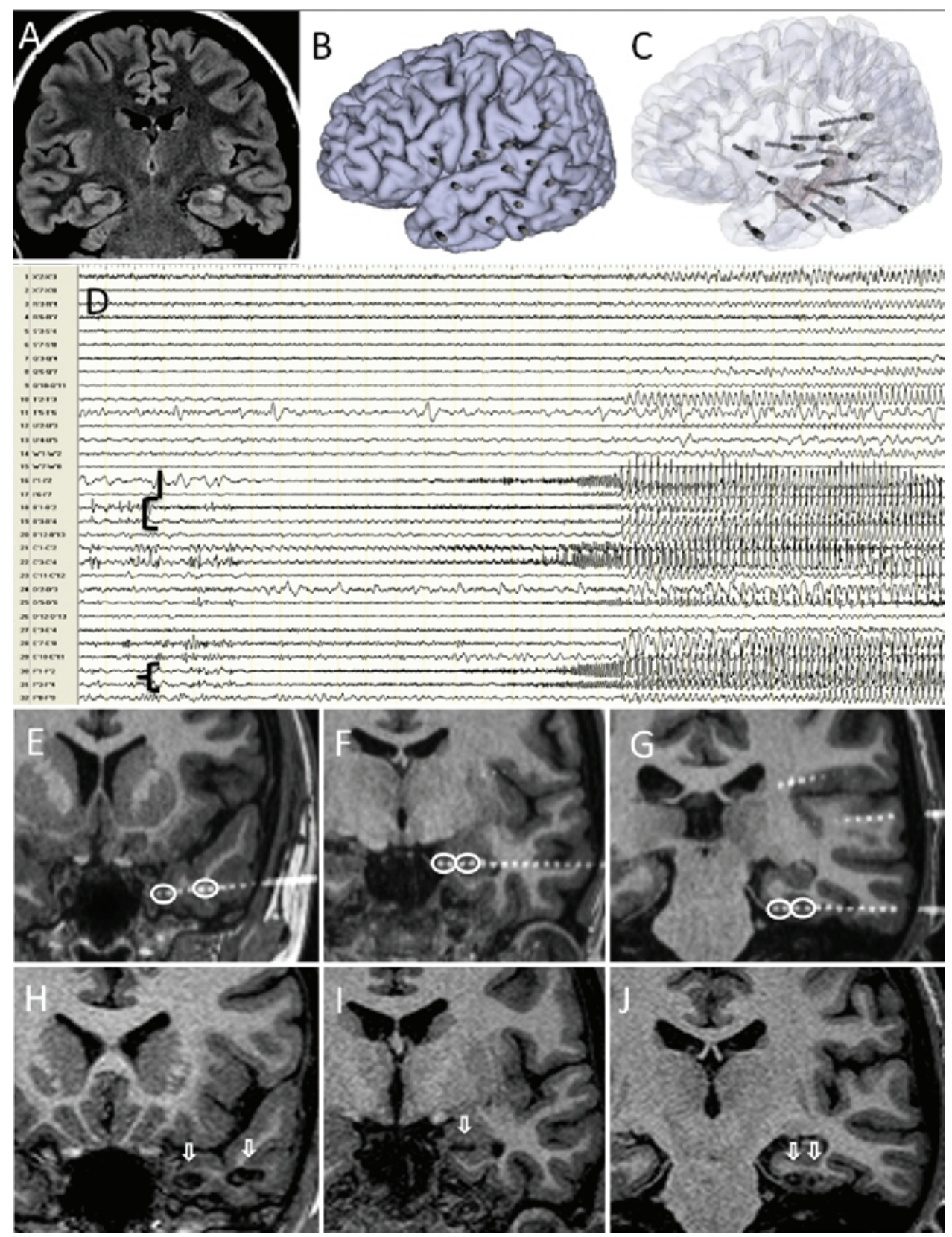

FIG. 2. Imaging from a 31-year-old woman with a history of febrile convulsions in infancy and onset of complex partial seizures at 8 years of age. A: This coronal MR image shows distinct hippocampal sclerosis, but some semiological features of the seizures (early speech disturbance, cough) and ictal scalp EEG findings suggested a possible extratemporal involvement in the ictal discharge. B and C: Perisylvian stereoelectroencephalographic exploration was performed. D-G: Ictal SEEG recording (D) revealed early-onset low-voltage fast activity in the mesial temporal pole (traces highlighted with vertical bar, recording contacts highlighted with circles in $\mathrm{E}$ ), in the anterior hippocampus (square bracket in D, circles in F), and in the mesiobasal temporal cortex (brace in D, circles in G). $\mathrm{H}-\mathrm{J}$ : An MR image acquired 6 months after RF-TC shows sites of coagulation in the temporal pole, hippocampus, and mesiobasal cortex (arrows). The patient was seizure free 1 year after the procedure, and antiepileptic medication was reduced. Figure is available in color online only. 
derwent surgery or according to MRI findings in patients who received only RF-TC, are reported in Table 4.

\section{Side Effects of and Morbidity After RF-TC}

In $11(12.3 \%)$ patients, a habitual seizure occurred during the coagulation procedure, in most cases while the coagulating regions in which electrical stimulations had induced similar ictal manifestations through SEEG evaluation. Four patients experienced fleeting local pain during coagulation of regions contiguous to the tentorium or to the cavernous sinus.

One patient presented with an unexpected, severe, and stable neurological deficit, presenting as a complex neuropsychological disorder. This 41-year-old man with nonlesional MRI results was evaluated by SEEG for possible left-posterior quadrant seizure onset (Fig. 3A and B), and he was considered at the end of SEEG monitoring to be a good candidate for resective surgery in the parietal lobe. However, he accepted RF-TC in the same area of eventual resection, and RF-TC of 23 sites in his left parietal lobe (according to the results of the SEEG recording) was performed. In the first weeks after the procedure, the patient developed severe reading and writing impairments and impairments in functions regarding numerical systems and calculation. A cranial CT scan revealed local brain swelling in the coagulated area, which was treated with steroids. Despite subsequent progressive clearing of the brain edema, the neuropsychological disturbances were still present 9 months after the procedure, and the patient had significant disability during work and in other daily activities. The patient is not seizure free, although a substantial decrease in seizure frequency and intensity has occurred, such that his resective procedure was cancelled and, at the time of writing, had not yet been performed. His postcoagulation MR images are shown in Fig. $3 \mathrm{C}-\mathrm{H}$.

One patient developed an expected and anticipated permanent motor deficit. This 28-year-old woman presented with disabling daily motor seizures that had occurred since the age of 9 years. Brain MRI revealed an FCD (presumably Type II) of the left precentral gyrus at the level of the hand primary motor area. The SEEG recording revealed overlapping between interictal and ictal electrical activity, the FCD, and the hand motor area. The high risk of permanent motor deficits with both open surgical resection and coagulation of her FCD were anticipated and discussed with the patient, who chose RF-TC as her first option. RF-TC of 7 areas at the FCD site was performed, and she immediately developed dense right hemiparesis, more evident at the distal segments of her left upper limb. After intensive physical rehabilitation, the patient was able to walk with support, and although recovery of the motor functions of her right hand was incomplete, she could drive a car. The patient, who at the time of this writing was still on antiepileptic drugs, was seizure free for 3 years postoperatively, and she subsequently presented 3 ictal manifestations in conjunction with decreased sodium blood levels (128 mEq/L).

Finally, a 15-year-old boy, who previously underwent 2 unsuccessful surgical resections of a Type II FCD in the right mesial premotor area, was submitted to an SEEG evaluation for seizure recurrence. According to the SEEG
TABLE 4. Etiologies of epilepsy in 89 patients*

\begin{tabular}{lc}
\hline \multicolumn{1}{c}{ Pathology } & No. of Pts \\
\hline Cryptogenic (no lesion) & 44 \\
\hline Nodular heterotopy & 12 \\
\hline FCD Type II & 9 \\
\hline Hippocampal sclerosis & 6 \\
\hline Glial scars & 6 \\
\hline Polymicrogyria & 4 \\
\hline FCD Type I & 2 \\
\hline Vascular malformation & 2 \\
\hline Band heterotopy & 2 \\
\hline Tuberous sclerosis & 2 \\
\hline
\end{tabular}

* Assessed as the best available evidence (histology for patients who underwent surgery and MRI findings for patients who received RF-TC as the only treatment).

findings, RF-TC of 2 sites just adjacent to the primary motor area of the left foot (as revealed by functional mapping) was performed. A transient motor deficit of the left foot appeared during the procedure, but complete recovery was achieved within several weeks. At the time of this writing, the patient had been seizure free for 3 years after treatment.

Therefore, unexpected, severe permanent neurological morbidity after RF-TC occurred in $1.1 \%$ (1 of 89 patients) of our cases.

\section{Discussion}

Results of this single-center retrospective study indicate that SEEG-guided RF-TC in the EZ may provide excellent results for seizures in patients with complex drug-resistant focal epilepsy, and seizure freedom or sustained worthwhile improvement was achieved in $18.0 \%$ and $10.1 \%$ of the patients, respectively.

\section{Pretreatment Evaluation}

Unlike most previous studies on stereotactic ablative techniques used to treat epilepsy, we report on a subpopulation of patients who all required invasive EEG recording, recommended for the particular complexity of their epilepsy. Indeed, apart from the pioneering reports on SEEGguided RF-TC by a group of authors in Lyon, France, ${ }^{4,12,13}$ recent studies on stereotactic TC have included patients with only noninvasive evaluation, ${ }^{7}$ a few patients who underwent invasive EEG, ${ }^{11,16,24}$ and individual patients who underwent local recording of electroencephalographic activity in the lesion targeted for coagulation. ${ }^{21,23}$

Therefore, because the seizure outcome of patients who require invasive EEG is generally less favorable, the contribution of RF-TC in controlling or improving seizures in our patients should be evaluated.

\section{Seizure Outcome After RF-TC}

Our results indicate that patients with a nodular heterotopy are the ideal candidates for RF-TC (8 [66.7\%] of 12 patients seizure free). As a matter of fact, nodular heter- 

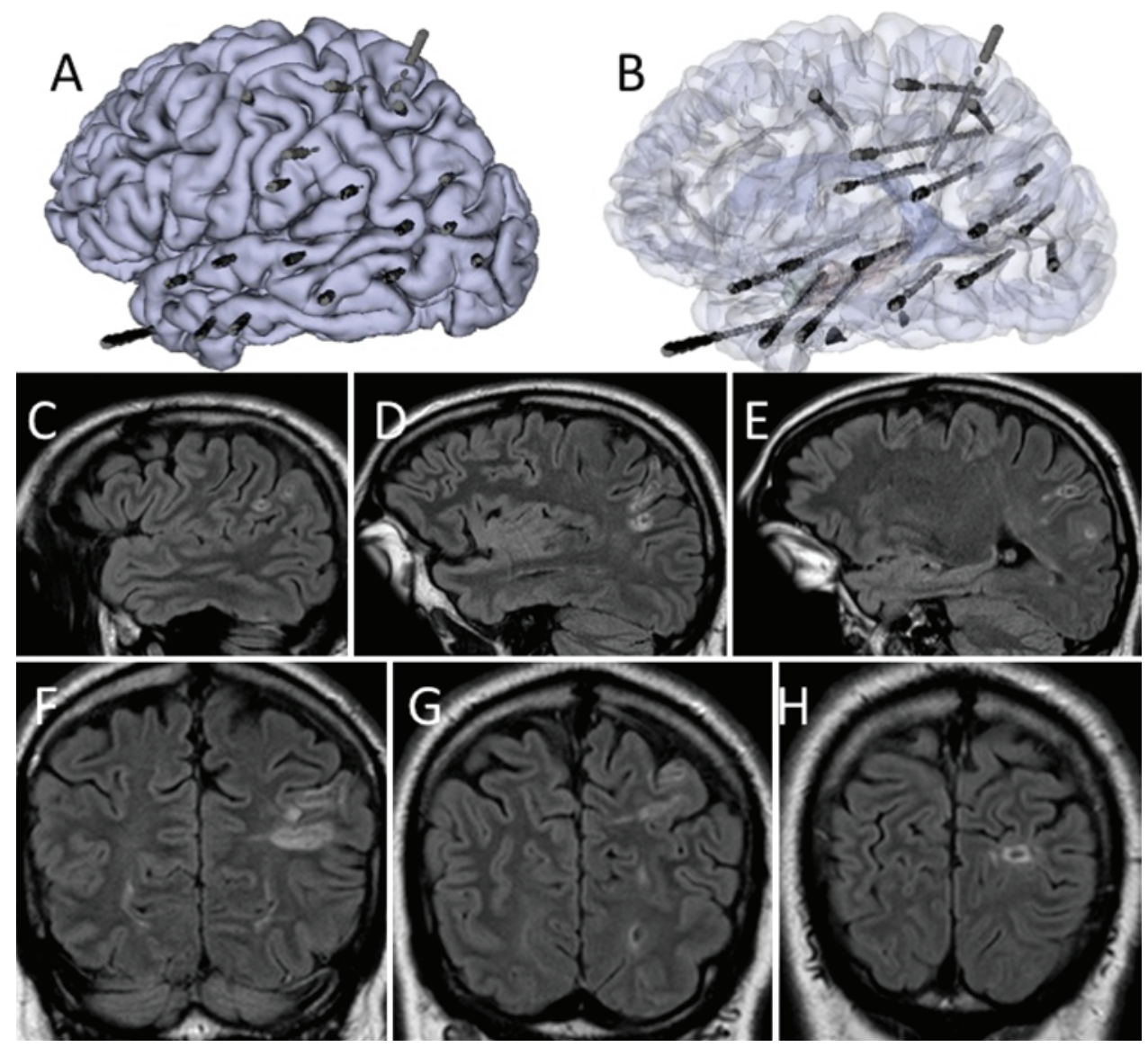

FIG. 3. A and B: A 41-year-old man was submitted to stereoelectroencephalographic exploration for drug-resistant epilepsy that presumably originated from the left posterior quadrant; no lesions were found with MRI. After coagulation of 23 sites in the parietal lobe, the patient developed a complex neuropsychological syndrome (see text). C-H: FLAIR MRI sequences in the sagittal (C-E) and coronal $(\mathrm{F}-\mathrm{H})$ planes obtained 6 months after RF-TC show some of the sites of coagulation. Figure is available in color online only.

otopies were recently treated by other groups using either RF-TC or laser TC., ${ }^{911,21}$ With time, as excellent results on seizures became evident in our experience, we were encouraged to offer RF-TC as the first option to patients with this etiology. Therefore, the strategy for electrode arrangement was implemented progressively for extensive coverage of the heterotopy to destroy the lesion as completely as possible, which enabled us to achieve good results not only in the less recent patients with a single nodule ${ }^{6}$ but also in those with bilateral (2 patients) or larger heterotopies. In particular, previous studies indicated that patients with bilateral heterotopies are not good candidates for surgical resection. ${ }^{22}$ Our results indicate that these patients should be considered for this potentially curative option.

Encouraging results have also been achieved in patients with hippocampal sclerosis, with $3(50 \%)$ of 6 patients seizure free after coagulation; only 3 of these patients had a follow-up time of $>12$ months and were available for statistical analysis. Excellent results after RF-TC have been reported for patients with mesial temporal lobe epilepsy (MTLE) and particularly in patients with hippocampal sclerosis..$^{14,16,17}$ These results, however, were obtained with extensive coagulation of the mesial temporal structures by electrodes placed along the major hippocampal axis.
In our patients, coagulation of the mesial structures, and specifically of the hippocampus, was limited to 1 or 2 sites, corresponding to the internal contacts of orthogonally placed electrodes. Additional targets for coagulation in these cases were the amygdala, the temporal pole, and the basal temporal cortex, according to indications from SEEG recording. Our patients with MTLE shared atypical clinical or electroencephalographic features that justified invasive SEEG recording to verify possible extratemporal implication of the EZ. We think, however, that RF-TC might be a valuable option also in more typical cases of MTLE when the risks involved with microsurgical resection are increased for age or for impaired general conditions.

Recently, RF-TC was used for the treatment of 2 patients with Type II FCD, and excellent results for seizures were achieved. ${ }^{23}$ In our experience, none of the 11 patients with an FCD (2 Type I and 9 Type II) achieved seizure freedom. A possible explanation is that we usually proceed to surgical resection with no invasive recording in patients with an FCD similar to the FCDs reported by Wellmer et al., ${ }^{23}$ whereas SEEG is reserved for patients with more extended cortical malformations, which are addressed less easily by focused RF-TC. 
Seizure outcomes were more favorable in lesional than in nonlesional cases $(25.6 \%$ and $10.9 \%$ of patients seizure free, respectively). All of our patients were imaged with 1.5-T MRI equipment. We cannot exclude the possibility that use of a 3-T MRI scanner could have revealed more subtle lesions undetected by our 1.5-T device, which would have enabled us to optimize the implantation strategy and increase the efficacy of RF-TC. Nevertheless, of 46 patients with no lesions seen with MRI, 5 were seizure free after RF-TC, and an additional 5 patients experienced worthwhile improvement. In these patients, SEEG guidance is essential for defining the EZ ${ }^{18}$ and identifying the sites in which coagulation should be performed. In some of our patients, coagulation might have interfered with complex epileptogenic networks at crucial nodes and thus hindered initiation of the ictal discharge and/or its spread through the symptomatogenic zone. To obtain this result, of course, a correct interpretation of the noninvasive findings in patients with no lesions seen with MRI is essential for adequately addressing the correct SEEG strategy.

A weak positive correlation was observed between a transient benefit from RF-TC and a Class I outcome after open surgery in patients who subsequently underwent surgery, although this finding was not statistically significant, probably because of the small sample size. However, this result suggests that the clinical effects of coagulation on seizures might have some prognostic value for subsequent resective surgery.

It may be argued that the impact on seizures observed after RF-TC in our patients resulted from the interference of intracerebral electrodes with the epileptic network. Nevertheless, in the same study period, we evaluated by SEEG 27 patients who were comparable to those in Group 2 and who refused RF-TC. No sustained change in either seizure frequency or the clinical pattern over time was observed in these cases (data not shown). Furthermore, in our series of $>600$ SEEG procedures, only 1 patient, investigated in 2002, has been seizure free since SEEG monitoring without any additional surgical interference. These data suggest that a postimplantation effect is a very unlikely explanation for the seizure outcomes observed in our RF-TC series.

\section{Side Effects and Complications}

The RF-TC procedures were basically well tolerated; painful sensations were reported occasionally when coagulation was performed close to dural structures. Also, the not-uncommon occurrence of habitual seizures during RF delivery was not a major problem.

During SEEG recording, thorough functional mapping was performed to minimize the risk of injury to critical areas and of permanent neurological deficits. This mapping also enabled us to discuss with 1 of our patients the predictable development of a motor deficit after RF-TC of her rolandic FCD.

The important neuropsychological syndrome developed by 1 patient after extensive coagulation of the dominant parietal lobe was probably a result of the confluence of several thermal lesions in a highly eloquent area, and it also would have been the likely result of open surgical resection of the same region. Functional mapping is very helpful in the localization of more elementary functions such as movement or sensation, but unfortunately it may fail to provide meaningful information about composite neuropsychological functions, such as those impaired in our patient. Indeed, site-by-site intracerebral electrical stimulations would be unlikely to result in clinical responses from such a complex functional network, which require simultaneous stimulations at multiple nodes.

The need to avoid thermal injury to eloquent areas and vascular structures indicates that in a number of cases, the coagulation plan proposed by the epileptologists after interpretation of the SEEG recording might not have been fully completed, thus leaving behind some areas that required treatment. This may explain some of the unfavorable outcomes in our patients but, at the same time, probably accounts for the safety of the procedure.

\section{Advantages and Limitations of the Technique}

The most obvious advantage of this technique is that it enables us to perform a potentially therapeutic stereotactic procedure with no additional placement of intracerebral devices besides those used for diagnostic SEEG recording. There are no additional costs for either consumables or prolonged hospitalization. Furthermore, SEEG recording provides both strong electroclinical evidence for guiding the treatment plan and functional information for minimizing neurological injuries.

An important disadvantage of the technique is the lack of real-time control of the progression of the lesion during the procedure. As a matter of fact, our preliminary laboratory experiences (unpublished data) suggested that modulating the progressive increase of current power affects the coagulated volume. For example, we noticed that slowing the increase of current power usually results in larger coagulation volumes, which may help when treating large-sized epileptogenic lesions. Conversely, thermal MRI sequences used for real-time control of coagulation have been implemented in the workflow of laser-induced thermal treatment of disorders of different body districts, including brain lesions and epilepsy. ${ }^{2}$ This process enables accurate planning of the thermal lesion, which can be tailored according to specific geometrical requirements with substantial safety margins. The development of instrumentations for RF-TC compatible with thermal MRI sequences is desirable for the future. Furthermore, future implementations of the technique might be achieved by evaluating how different procedural parameters (including, e.g., time of RF delivery, number of coagulation sites, speed of power increase, and differences in coagulated substrates) affect the anatomical results of TC as revealed by either postthermocoagulation MRI or histological examination of surgical specimens.

\section{Limitations of the Study}

The results presented here derive from a single-center retrospective study, and we acknowledge this as the main limitation. Furthermore, an additional limitation of the study is a very likely selection bias. Indeed, our case series included a proportion of patients with nodular heterotopies $(13.5 \%)$ that undoubtedly exceeds that expected in a surgical series of patients with drug-resistant focal epilepsy. As 
a matter of fact, as our experience with SEEG-guided RFTC increased, it became clear to us that patients with nodular heterotopies are optimal candidates for this treatment option, so the preliminary results on the excellent outcome obtained for 5 of these patients were recently published. ${ }^{6}$ Therefore, because the decision to accept RF-TC was made by the patients or their guardians after a thorough discussion with the epileptologists and the neurosurgeons of the team, it is likely that we had a more optimistic attitude when we offered this treatment to patients with nodular heterotopies than to patients with other etiologies. Similar reasons probably explain the considerable rate $(24.7 \%)$ of patients excluded from surgery after SEEG evaluation in this series, which is higher than that recorded in our whole population of patients who underwent SEEG (approximately $10 \%{ }^{5}$ ). In fact, it is likely that a number of patients amenable to resective surgery after SEEG, worried by the idea of having their brain "burned," had refused RF-TC in favor of a more traditional and potentially curative option. Conversely, patients with no chance to benefit from a resective procedure might have been more positive toward the proposed option as their one and only, although palliative, chance. It must be stressed, however, that 5 good candidates, worried by the possible risks of open surgery, refused the recommended resection and accepted RF-TC as their only treatment.

Our results, therefore, need to be confirmed by a hopefully prospective multicenter study. Furthermore, a long-term evaluation of seizure outcomes is required to ascertain if RF-TC can provide stable and long-lasting inactivation of the EZ or if otherwise reorganization of epileptogenic networks may occur after their temporary suppression provided by thermal lesioning. An important implication of this unresolved issue is that it is unclear if medical treatment can be tapered and stopped in seizurefree patients according to the same criteria adopted for those who undergo microsurgical resection.

\section{Conclusions}

Stereoelectroencephalography-guided RF-TC is a relatively safe treatment option for patients with complex drug-resistant focal epilepsy that requires invasive EEG evaluation. A small, but not negligible, subset of our patients achieved seizure freedom or worthwhile improvement, enabling them to indefinitely defer open surgery or to benefit from an otherwise unexpected treatment option when they are not amenable to surgical resection.

\section{Acknowledgments}

We acknowledge the EEG laboratory technicians (Antonio Menna, Maurizio Rossi, Katrina Sambusida, Fabrizio Sandrin, and Claudia Vilasi) for their invaluable help in intensive video-SEEG monitoring of our patients.

\section{References}

1. Bancaud J, Talairach J, Bonis A, Schaub C, Szikla G, Morel P, et al: La Stéréo-Électroencéphalographie dans l'Épilepsie. Paris: Masson, 1965
2. Bazrafshan B, Hübner F, Farshid P, Hammerstingl R, Paul J, Vogel V, et al: Temperature imaging of laser-induced thermotherapy (LITT) by MRI: evaluation of different sequences in phantom. Lasers Med Sci 29:173-183, 2014

3. Cardinale F, Cossu M, Castana L, Casaceli G, Schiariti MP, Miserocchi A, et al: Stereoelectroencephalography: surgical methodology, safety, and stereotactic application accuracy in 500 procedures. Neurosurgery 72:353-366, 2013

4. Catenoix H, Mauguière F, Guénot M, Ryvlin P, Bissery A, Sindou M, et al: SEEG-guided thermocoagulations: a palliative treatment of nonoperable partial epilepsies. Neurology 71:1719-1726, 2008

5. Cossu M, Cardinale F, Castana L, Citterio A, Francione S, Tassi L, et al: Stereoelectroencephalography in the presurgical evaluation of focal epilepsy: a retrospective analysis of 215 procedures. Neurosurgery 57:706-718, 2005

6. Cossu M, Fuschillo D, Cardinale F, Castana L, Francione S, Nobili L, et al: Stereo-EEG-guided radio-frequency thermocoagulations of epileptogenic grey-matter nodular heterotopy. J Neurol Neurosurg Psychiatry 85:611-617, 2014

7. Curry DJ, Gowda A, McNichols RJ, Wilfong AA: MR-guided stereotactic laser ablation of epileptogenic foci in children. Epilepsy Behav 24:408-414, 2012

8. Engel J Jr, Van Ness PC, Rasmussen TB: Outcome with respect to epileptic seizures, in Engel J Jr (ed): Surgical Treatment of the Epilepsies, ed 2. New York: Raven Press, 1993, pp 609-621

9. Esquenazi Y, Kalamangalam GP, Slater JD, Knowlton RC, Friedman E, Morris SA, et al: Stereotactic laser ablation of epileptogenic periventricular nodular heterotopia. Epilepsy Res 108:547-554, 2014

10. Gonzalez-Martinez J, Mullin J, Vadera S, Bulacio J, Hughes G, Jones S, et al: Stereotactic placement of depth electrodes in medically intractable epilepsy. J Neurosurg 120:639-644, 2014

11. Gonzalez-Martinez J, Vadera S, Mullin J, Enatsu R, Alexopoulos AV, Patwardhan R, et al: Robot-assisted stereotactic laser ablation in medically intractable epilepsy: operative technique. Neurosurgery 10 (Suppl 2):167-173, 2014

12. Guénot M, Isnard J, Catenoix H, Mauguière F, Sindou M: SEEG-guided RF-thermocoagulation of epileptic foci: a therapeutic alternative for drug-resistant non-operable partial epilepsies. Adv Tech Stand Neurosurg 36:61-78, 2011

13. Guénot M, Isnard J, Ryvlin P, Fischer C, Mauguière F, Sindou M: SEEG-guided RF thermocoagulation of epileptic foci: feasibility, safety, and preliminary results. Epilepsia 45:1368-1374, 2004

14. Kalina M, Lisck R, Vojtech Z, Adámková E, Procházka T, Marecková I, et al: Stereotactic amygdalohippocampectomy for temporal lobe epilepsy: promising results in 16 patients. Epileptic Disord 9 (Suppl 1):S68-S74, 2007

15. Kameyama S, Murakami H, Masuda H, Sugiyama I: Minimally invasive magnetic resonance imaging-guided stereotactic radiofrequency thermocoagulation for epileptogenic hypothalamic hamartomas. Neurosurgery 65:438-449, 2009

16. Liscak R, Malikova H, Kalina M, Vojtech Z, Prochazka T, Marusic P, et al: Stereotactic radiofrequency amygdalohippocampectomy in the treatment of mesial temporal lobe epilepsy. Acta Neurochir (Wien) 152:1291-1298, 2010

17. Malikova H, Vojtech Z, Liscak R, Prochazka T, Vymazal J, Vladyka V, et al: Stereotactic radiofrequency amygdalohippocampectomy for the treatment of mesial temporal lobe epilepsy: correlation of MRI with clinical seizure outcome. Epilepsy Res 83:235-242, 2009

18. McGonigal A, Bartolomei F, Régis J, Guye M, Gavaret M, Trébuchon-Da Fonseca A, et al: Stereoelectroencephalography in presurgical assessment of MRI-negative epilepsy. Brain 130:3169-3183, 2007

19. Munari C, Kahane P, Tassi L, Francione S, Hoffmann D, Lo 
Russo G, et al: Intracerebral low frequency electrical stimulation: a new tool for the definition of the "epileptogenic area"? Acta Neurochir Suppl (Wien) 58:181-185, 1993

20. Parrent AG, Blume WT: Stereotactic amygdalohippocampotomy for the treatment of medial temporal lobe epilepsy. Epilepsia 40:1408-1416, 1999

21. Schmitt FC, Voges J, Buentjen L, Woermann F, Pannek HW, Skalej M, et al: Radiofrequency lesioning for epileptogenic periventricular nodular heterotopia: a rational approach. Epilepsia 52:e101-e105, 2011

22. Tassi L, Colombo N, Cossu M, Mai R, Francione S, Lo Russo G, et al: Electroclinical, MRI and neuropathological study of 10 patients with nodular heterotopia, with surgical outcomes. Brain 128:321-337, 2005

23. Wellmer J, Kopitzki K, Voges J: Lesion focused stereotactic thermo-coagulation of focal cortical dysplasia IIB: a new approach to epilepsy surgery? Seizure 23:475-478, 2014

24. Willie JT, Laxpati NG, Drane DL, Gowda A, Appin C, Hao $\mathrm{C}$, et al: Real-time magnetic resonance-guided stereotactic laser amygdalohippocampotomy for mesial temporal lobe epilepsy. Neurosurgery 74:569-585, 2014

\section{Author Contributions}

Conception and design: Cossu, Tassi. Acquisition of data: Cossu, Fuschillo, Casaceli, Pelliccia, Castana. Analysis and interpretation of data: Cossu, Fuschillo, Casaceli, Pelliccia, Nobili, Cardinale. Drafting the article: Cossu. Critically revising the article: all authors. Reviewed submitted version of manuscript: all authors. Approved the final version of the manuscript on behalf of all authors: Cossu. Statistical analysis: Cardinale. Study supervision: Cossu.

\section{Correspondence}

Massimo Cossu, Epilepsy Surgery Center, Department of Neuroscience, Niguarda Hospital, Piazza Ospedale Maggiore 3, 20162 Milan, Italy.email: massimo.cossu@ospedaleniguarda.it. 